\title{
Avaliação da Efetividade da Reserva Biológica do Gurupi na Conservação de Vertebrados Terrestres de Médio e Grande Porte
}

\author{
Eloisa Neves Mendonça ${ }^{1,2}$, Alexandre Martins Costa Lopes ${ }^{3,4}$, Ana Luísa K. M. Albernaz ${ }^{5}$ \& Elildo A. R. Carvalho Jr.6,7
}

Recebido em 09/09/2020 - Aceito em 27/04/2021

1 Museu Paraense Emilio Goeldi/MPEG, Programa de Pós-graduação em Biodiversidade e Evolução/PPGBE, Belém/PA, Brasil. CEP: 66.077-530. <eloisa.mendonca@icmbio.gov.br>.

2 Instituto Chico Mendes de Conservação da Biodiversidade/ICMBio, Reserva Biológica do Gurupi, Açailandia/MA, Brasil. CEP: 65.930-000. <eloisa.mendonca@icmbio.gov.br>.

3 Instituto de Pesquisa e Conservação de Tamaduás no Brasil, Parnaíba/PB, Brasil. CEP: 64.200-025. <alexandre@tamandua.org>.

4 Universidade Federal do Piauí, Programa de Pós-graduação em Biodiversidade e Conservação. Brasil. <alexandre@tamandua.org>.

${ }_{5}$ Museu Paraense Emilio Goeldi/MPEG, Coordenação de Ciências da Terra e Ecologia, Belém/PA, Brasil. CEP: 66.077-530. <anakma@museu-goeldi.br>.

6 Instituto Chico Mendes de Conservação da Biodiversidade/ICMBio, Centro Nacional de Pesquisa e Conservação de Mamíferos Carnívoros/CENAP, Atibaia/SP, Brasil. CEP: 12.952-011. <elildo.carvalho-junior@icmbio.gov.br>.

7 Faculty of Ecology and Natural Resource Management, Norwegian University of Life Sciences, P.O. Box 5003, 1432, Ås, Norway. <elildo.carvalho-junior@icmbio.gov.br>.

RESUMO - O protocolo de armadilhamento fotográfico TEAM para o monitoramento de vertebrados terrestres de médio e grande porte permite estimativas de parâmetros da biodiversidade que informam os gestores de áreas protegidas sobre os impactos de ameaças ou de ações de manejo da biodiversidade. Neste trabalho, apresentamos resultados de 4 anos do monitoramento TEAM na Reserva Biológica do Gurupi com objetivo de avaliar sua efetividade na conservação de vertebrados terrestres de médio e grande porte da Amazônia maranhense. Foram avaliadas tendências temporais na riqueza e composição da comunidade, nas taxas de ocupação das espécies monitoradas e no Wildlife Picture Index, índice sensível às mudanças na biodiversidade. Foram registradas 36 espécies de vertebrados de médio e grande porte, representando $72 \%$ das aves e $96 \%$ dos mamíferos com ocorrência na Amazônia maranhense. As populações monitoradas, inclusive de espécies endêmicas e ameaçadas de extinção, permaneceram estáveis ao longo de 4 anos de monitoramento. A taxa de ocupação da maioria das espécies aumentou no período de monitoramento, refletindo no aumento do Wildlife Picture Index. Possivelmente essas tendências têm relação com o grande incêndio florestal que assolou a Reserva Biológica do Gurupi em 2015, indicando a recuperação da biota e a resiliência frente a eventos extremos. Os resultados obtidos demonstram que a Reserva Biológica do Gurupi tem sido efetiva e tem papel de destaque na conservação de vertebrados terrestres de médio e grande porte da Amazônia maranhense.

Palavras-chave: Amazônia maranhense; protocolo TEAM; monitoramento da biodiversidade.

\section{Evaluating effectiveness of Gurupi Biological Reserve of the medium-to large-sized Terrestrial Vertebrates Conservation}

\begin{abstract}
The TEAM camera trap protocol to monitor medim- to large-sized terrestrial vertebrates, allow estimates of biodiversity parameters that inform protected area managers about threats to biodiversity or the impact of management actions. In this study, we present results of four years of camera trap monitoring at Gurupi Biological Reserve to evaluate its effectiveness in conserving medium- to large-sized terrestrial vertebrates from the Amazon region of Maranhão Brazilian State. We evaluate trends in species richness, community composition, species occupancy rates, and Wildlife Picture Index to assess biodiversity status and trends. We recorded 36 species of medium- to largesized vertebrates, representing $72 \%$ of the birds and $96 \%$ of the mammals of Amazon region of Maranhão. The monitored populations, including endemic and endangered species, remained stable over 4 years monitoring. Occupancy rates for most species increased as well as the Wildlife Picture Index. Possibly these trends are related to wildlife recovery after a wildfire that hit Gurupi Biological Reserve in 2015, and indicates the resilience of the protected area after a severe impact. The results
\end{abstract}


show the Gurupi Biological Reserve has been effective in its goal of conserving medium-to large-sized terrestrial vertebrates.

Keywords: Amazon region of Maranhão Brazilian State; TEAM protocol; biodiversity monitoring.

\title{
Evaluación de la Efectividad de la Reserva Biológica del Gurupi en la Conservación de Vertebrados Terrestres de Mediano y Gran Tamaño
}

\begin{abstract}
RESUMEN - El protocolo de captura fotográfica TEAM para monitorear vertebrados terrestres medianos y grandes, permite estimaciones de parámetros de biodiversidad que informan a los administradores de áreas protegidas sobre los impactos de las amenazas o acciones de gestión de la biodiversidad. En este trabajo, presentamos los resultados de 4 años de monitoreo TEAM en Reserva Biológica del Gurupi con el objetivo de evaluar su efectividad en la conservación de vertebrados terrestres medianos y grandes en la región amazónica el estado brasileño del Maranhão. Fueram evaluadas las tendencias temporales en la riqueza y composición de la comunidad, en las tasas de ocupación de las especies monitoreadas y en el Wildlife Picture Index, un índice sensible a los cambios en la biodiversidad. Fueram registradas 36 especies que representan $72 \%$ de las aves y $96 \%$ de los mamíferos presentes en la región amazónica del Maranhão. Las poblaciones monitoreadas, incluidas las especies endémicas y en peligro de extinción, se mantuvieron estables durante 4 años de monitoreo. La tasa de ocupación de la mayoría de las especies tuvo un incremento, tan bien el Wildlife Picture Index. Posiblemente estas tendencias estén relacionadas con el gran incendio forestal que afectó la Reserva Biológica del Gurupi en 2015, indicando la recuperación de la biota y la resiliencia de la unidad de conservación frente a eventos extremos. Los resultados obtenidos demuestran que la Reserva Biológica del Gurupi ha sido eficaz y tiene un papel destacado en la conservación de vertebrados terrestres medianos y grandes en la región amazónica del Maranhão.
\end{abstract}

Palabras clave: Amazonia el Estado brasileño del Maranhão; protocolo TEAM; monitoreo de la biodiversidad.

\section{Introdução}

O monitoramento da biodiversidade é uma atividade essencial para informar sobre desvios dos sistemas biológicos em relação ao estado desejado, mensurar o sucesso de ações de manejo e detectar o efeito de perturbações ambientais (Legg \& Nagy, 2006). Programas de monitoramento eficazes requerem bons indicadores, uma definição prévia, ou ao menos uma noção aproximada, do grau de variação aceitável desses indicadores (O'Brien \& Kinnaird, 2013; Beaudrot et al., 2018), e desenhos amostrais que permitam a detecção de mudanças significativas no estado da biodiversidade, ou seja, desenhos com poder estatístico adequado (Fairweather, 1991; Legg \& Nagy, 2006; Field et al., 2007).

Responsável pela gestão das unidades de conservação federais, o Instituto Chico Mendes de Conservação da Biodiversidade (ICMBio), instituiu, em 2017, o Programa Nacional de Monitoramento da Biodiversidade (Programa Monitora), voltado ao monitoramento do estado da biodiversidade e serviços ecossistêmicos associados, como subsídio à avaliação da efetividade de conservação do sistema de unidades de conservação, à adaptação às mudanças climáticas e ao uso e manejo das unidades de conservação, bem como às estratégias de conservação das espécies ameaçadas de extinção em todo o território nacional (ICMBio, 2017; Monitora et al., 2018).

O Monitora adotou o protocolo TEAM de armadilhamento fotográfico (Jansen et al., 2014) como protocolo avançado para o monitoramento de vertebrados terrestres de médio e grande porte. Concebido pela rede TEAM (Tropical Ecology Assessment and Monitoring network), esse protocolo foi desenvolvido com o objetivo de viabilizar a detecção de tendências temporais na biodiversidade e a identificação de efeitos advindos de mudanças climáticas e no uso do solo (Ahumada et al., 2016). Os vertebrados terrestres de médio e grande porte possuem especial importância ecológica, econômica e estética (Ahumada et al., 2016; Lacher et al., 2019), e são particularmente vulneráveis aos impactos das atividades humanas, como perda e degradação de habitat, superexploração e mudanças climáticas (Schipper et al., 2008; Dirzo et al., 2014). 
Além de inventariar a comunidade de vertebrados terrestres de médio e grande porte, a alta qualidade dos dados e o desenho amostral robusto do protocolo TEAM, permitem estimativas de diversos parâmetros de biodiversidade. No nível de espécie, os modelos de ocupação permitem inferências sobre tendências populacionais ao longo do tempo (MacKenzie \& Nichols, 2004, Ahumada et al., 2011, 2013), e sobre a influência de variáveis ambientais ou impactos antropogênicos no uso do espaço (Rovero \& Ahumada, 2017). No nível da comunidade, as análises permitem estimar a riqueza e composição de espécies e suas variações temporais e espaciais (Rovero \& Ahumada, 2017).

Contudo o principal índice de biodiversidade adotado pelo protocolo TEAM é o WPI - Wildlife Picture Index (O'Brien \& Kinnaird, 2013), desenvolvido especificamente para avaliar mudanças na riqueza e ocupação das espécies na comunidade, a partir de dados de armadilhas fotográficas (O'Brien et al., 2010). O WPI é sensível às mudanças na biodiversidade e atende aos requisitos da Convenção da Diversidade Biológica, podendo informar os gestores de áreas protegidas sobre o impacto de ameaças ou sobre a efetividade de ações de manejo sobre a biodiversidade (Ahumada et al., 2013, 2016).

Dentre as unidades de conservação (UCs) geridas pelo ICMBio, a Reserva Biológica do Gurupi (REBIO do Gurupi) vem monitorando vertebrados terrestres por meio do protocolo TEAM desde 2016. Com objetivo de avaliar a efetividade da REBIO do Gurupi em relação ao seu objetivo de criação, de conservar a biodiversidade da região, neste trabalho apresentamos o inventário de vertebrados terrestres de médio e grande porte da REBIO do Gurupi; analisamos a variação temporal na riqueza e ocupação das espécies alvo do monitoramento, com destaque para espécies ameaçadas de extinção; e estimamos o WPI da comunidade ao longo do monitoramento.

\section{Área de estudo}

A REBIO do Gurupi (-0346's e -46²' O) foi criada com objetivo de preservar amostra representativa da diversidade biológica da Amazônia maranhense (Brasil, 1988). A UC tem uma área de 271 mil ha e conforma com as terras indígenas vizinhas um mosaico de áreas protegidas com mais de 2 milhões de hectares, constituindo o principal remanescente de floresta Amazônica no Maranhão (Celentano et al., 2017; Figura 1) e na Área de Endemismo Belém, a mais ameaçada pelo desmatamento dentre as áreas de endemismos da Amazônia (Almeida \& Vieira, 2010; Braz et al., 2017). A REBIO do Gurupi tem grande número de espécies de destacado valor para a conservação, das quais 46 espécies de mamíferos e aves endêmicas e/ou ameaçadas de extinção (Carvalho Jr., 2012; Lima et al, 2015; Buss et al., 2017; Carvalho Jr., 2017; Alteff et al., 2019), com destaque para as espécies endêmicas Cebus kaapori, Chiropotes satanas, Psophia obscura e Crax fasciolata pinima, com status de criticamente ameaçadas de extinção, de acordo com a lista oficial brasileira (MMA, 2014).

AREBIO do Gurupi está localizada em região de clima tropical com inverno seco, temperatura média anual acima de $26^{\circ} \mathrm{C}$ e a precipitação no mês mais seco abaixo de $60 \mathrm{~mm}$ (Alvares et al., 2013)

A vegetação é característica da Amazônia de terra firme (Braga, 1979), classificada de acordo com IBGE (2004) como floresta ombrófila densa. $\mathrm{Na}$ composição florística espécies de interesse econômico para a indústria madeireira, como ipê (Tabebuia spp), angelim (Andira sp, Dinizia sp e Hymenolobium spp), maçaranduba (Manilkara spp), jatobá (Hymenaea spp), e pauóleo (Copaifera spp) dentre outras, são alvos da extração ilegal de madeira desde a criação da unidade de conservação (IBAMA, 1999; Martins \& Oliveira, 2011).

Nas últimas três décadas a exploração ilegal de madeira, a ocupação humana e a pecuária levaram a uma perda de $30 \%$ na cobertura florestal da REBIO do Gurupi (Hessel \& Lisboa, 2015; Celentano et al., 2017). Além disso, a REBIO é frequentemente afetada por incêndios florestais decorrentes das atividades humanas ainda existentes em seus limites, e em 2015 os incêndios florestais atingiram aproximadamente $50 \%$ de sua área.

O monitoramento TEAM na REBIO do Gurupi tem sido realizado no setor norte, onde houve exploração ilegal de madeira de 1990 até 2011, quando a gestão da REBIO do Gurupi implementou uma base de proteção permanente em local estratégico que levou à completa interrupção da exploração ilegal madeireira (Carvalho Jr. et al., 2020). 


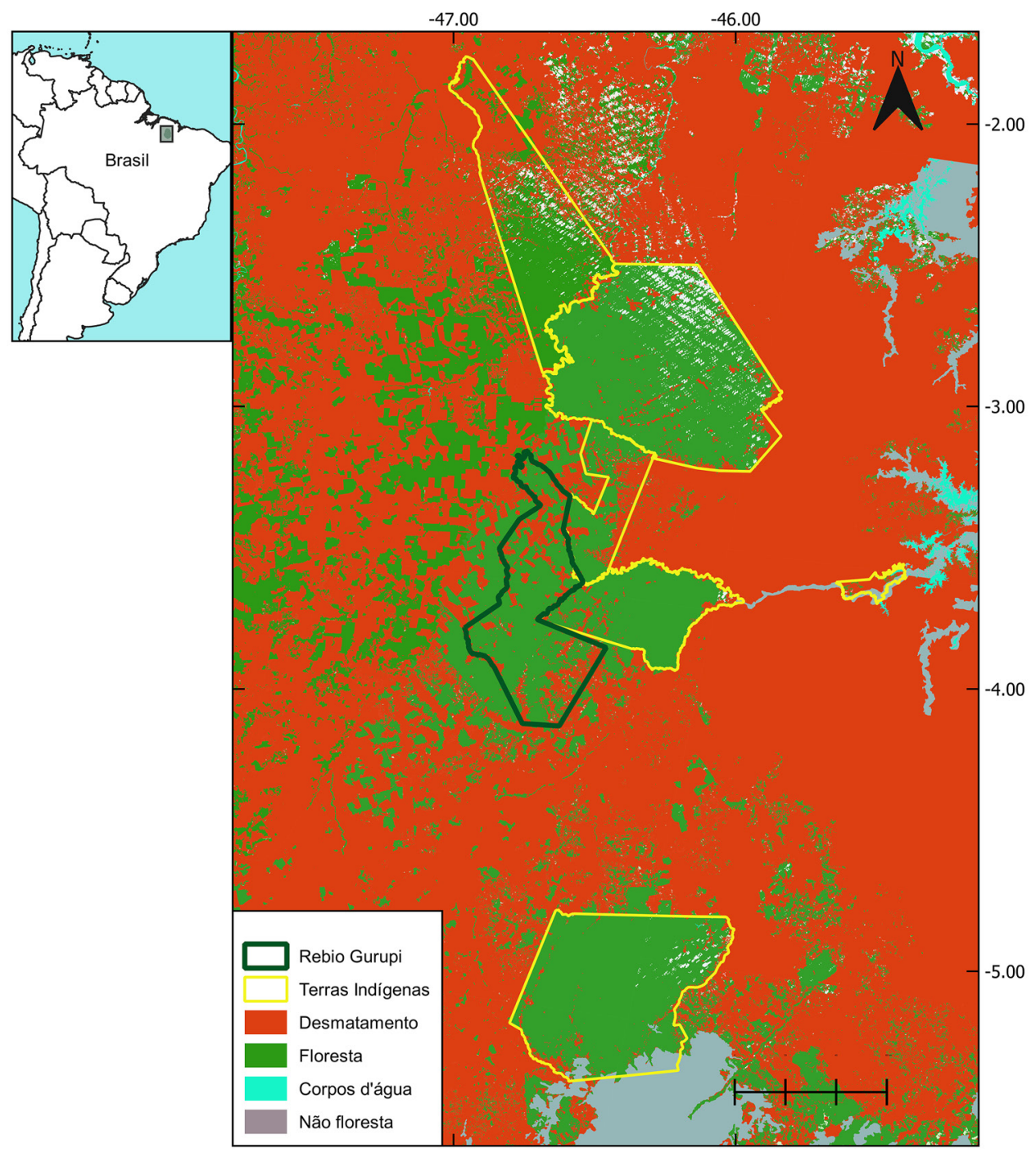

Figura 1 - Reserva Biológica do Gurupi e terras indígenas vizinhas no contexto do desmatamento da Amazônia Maranhense. Fonte: http://www.inpe.br/cra/projetos_pesquisas/terraclass2014.php.

\section{Metodologia}

As amostragens seguiram o protocolo TEAM para vertebrados terrestres (TEAM Network, 2011). Foram definidos dois blocos de amostragem, em áreas de floresta distantes pelo menos $500 \mathrm{~m}$ das estradas internas da UC. No total, foram estabelecidas 61 estações de armadilhamento fotográfico, numa grade regular com densidade média de uma estação a cada $2 \mathrm{~km}^{2}$, resultando numa área amostrada mínima de $120 \mathrm{~km}^{2}$ (Figura 2). As armadilhas fotográficas (Bushnell, modelo Trophy Cam) foram instaladas anualmente durante a estação seca, onde permaneceram funcionando continuamente no mínimo 30 dias. Não foram usadas iscas para atrair os animais.
Triagem das imagens - As imagens das armadilhas fotográficas foram processadas no software wild.ID, seguindo a IUCN Red List como autoridade taxonômica (Fegraus et al., 2011). Foi assumido o intervalo de 60 minutos entre fotos da mesma espécie na mesma câmera, para garantir a independência entre os eventos de detecção (Sollman, 2018).

As análises foram direcionadas para vertebrados terrestres de médio e grande porte passíveis de monitoramento com armadilhas fotográficas. Portanto, foram considerados somente os registros de mamíferos terrestres $>1 \mathrm{~kg}$ de massa corporal e as aves terrestres cinegéticas $>0,3 \mathrm{~kg}$ das famílias Cracidae, Odontophoridae, Psophiidae e Tinamidae. A referência da massa 


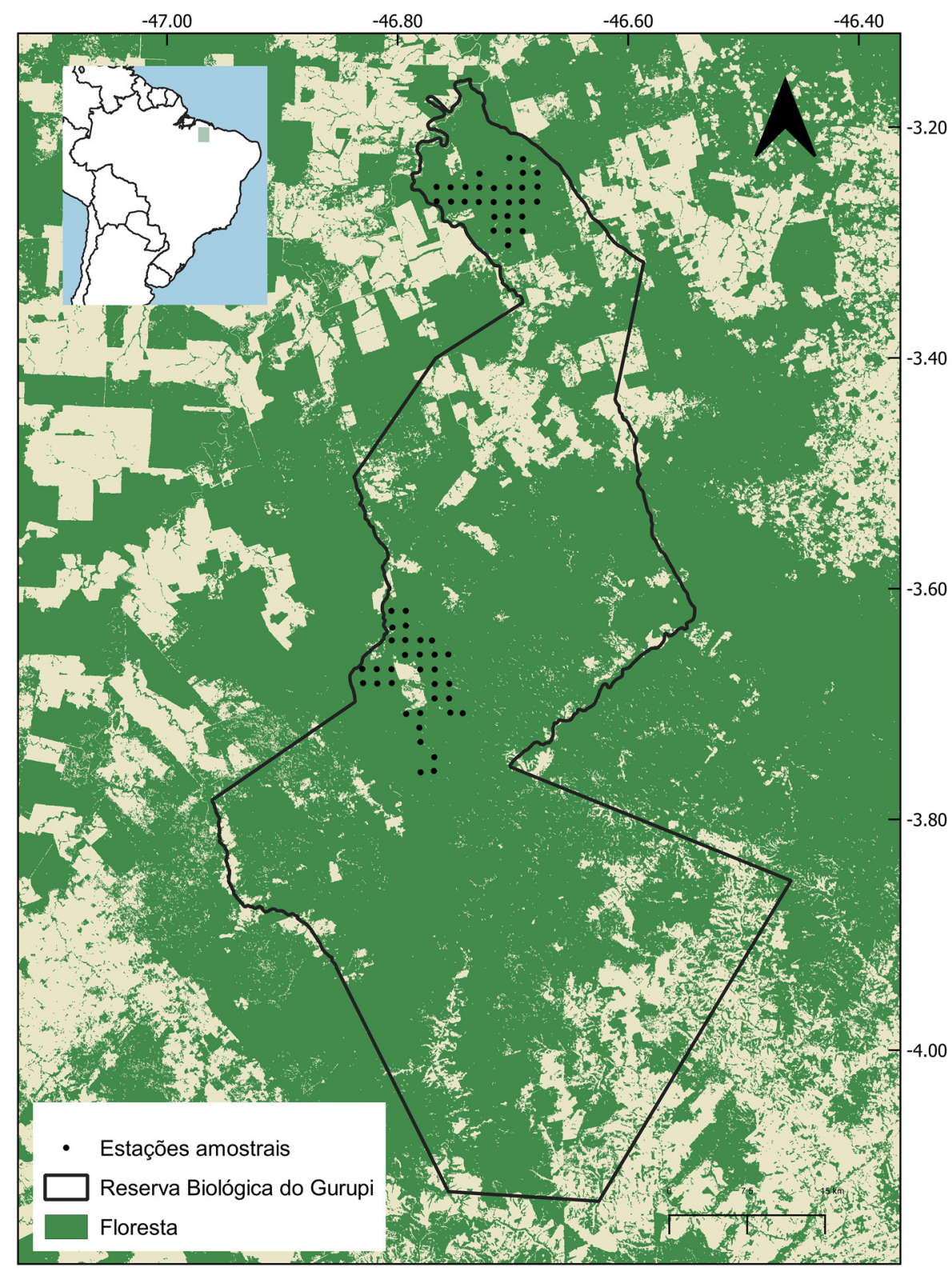

Figura 2 - Estações amostrais do monitoramento TEAM na REBIO do Gurupi. Fonte: http://mapbiomas.org.

corporal das espécies foi obtida de Wilman et al. (2014). Devido à dificuldade de identificação, as espécies dos gêneros Crypturellus, Dasypus, Leopardus, Mazama, Penelope e Tinamus foram agrupadas para as análises de WPI e ocupação.

Análises dos dados - A composição e riqueza de espécies foram analisadas a partir do inventário das espécies registradas pelo monitoramento e de sua comparação com as listas de espécies de ocorrência na Amazônia maranhense apresentadas em Oren \& Roma (2011), Lima et al. (2015), Vieira \& Oliveira
(2020), permitindo avaliar a representatividade da UC em relação a riqueza de vertebrados terrestres de médio e grande porte da região.

Para analisar se houve mudança na riqueza de espécies ao longo do tempo, foram estimados para cada ano de monitoramento, os índices de riqueza pelo estimador jackknife1 (Burnham \& Overton, 1978, 1979), com intervalo de confiança de $95 \%$ calculados com o pacote Vegan (Oksanen et al., 2019) em ambiente $\mathrm{R}$ ( $\mathrm{R}$ Development Core Team, 2019).

Por meio do modelo dinâmico de MacKenzie et al. (2003), foi analisada a variação 
temporal nas taxas de ocupação das espécies alvo do monitoramento. Esta taxa é uma medida de distribuição espacial, que estima a probabilidade de uma determinada área estar ocupada pela espécie, levando em consideração a probabilidade de detecção da espécie (MacKenzie et al., 2002). A partir de uma matriz com os dados de registros, de detecção e não detecção, de cada espécie, em cada estação amostral $e$ em cada ano de amostragem, o modelo utiliza a probabilidade de detecção estimada para cada local, para calcular a probabilidade de a espécie estar presente (Ahumada et al., 2016). O modelo dinâmico considera ainda as probabilidades de extinção e de colonização entre os diferentes anos, parâmetros importantes para entender a dinâmica populacional das espécies (O'Brien \& Kinnaird, 2013).

O modelo dinâmico de ocupação multiespécies foi gerado utilizando abordagem Bayesiana com o software JAGS (Plummer, 2003), executado no pacote R2jags (Yu-Sung, 2020) em ambiente R (R Development Core Team, 2019). $\mathrm{O}$ modelo foi rodado com três cadeias de Monte Carlo via Cadeias de Markov com 1100 iterações, período de aquecimento (burn-in) de 100 e thinning de 100.

O modelo dinâmico multi-espécies gerou taxas de ocupação para as espécies, para todos os anos de amostragem. O WPI foi calculado como a média geométrica da taxa de ocupação de todas as espécies na comunidade, escalonado pela ocupação no primeiro ano de amostragem (2016), que serve como linha de base (O'Brien \& Kinnaird, 2013).

Para avaliar se houve mudanças significativas no WPI e na taxa de ocupação de espécies ao longo do tempo, foi considerado um nível de significância de $10 \%$. O relaxamento do nível de significância $(10 \%$ ao invés dos convencionais $5 \%$ ) se justifica pelo ganho no poder estatístico e na capacidade de detectar declínios populacionais antecipadamente (Field et al., 2007). Na conservação de espécies ameaçadas, os custos resultantes de erros do tipo II (não detectar declínios caso estejam ocorrendo) tendem a ser maiores do que os resultantes de erros do tipo I (detectar declínios sem que estejam de fato ocorrendo) (Field et al., 2007; Beaudrot et al., 2018).

O modelo de ocupação e o WPI foram calculados no programa $\mathrm{R}$ ( $\mathrm{R}$ Development Core Team, 2019), seguindo as especificações de (O'Brien \& Kinnaird, 2013), a partir de códigos gentilmente cedidos por Jorge Ahumada (Conservation International).

\section{Resultados}

Os quatro anos de monitoramento resultaram num esforço amostral de 13.020 armadilhas-dia e 114.199 fotos de animais (Tabela 1). Considerando somente os vertebrados terrestres de médio e grande porte que atenderam aos critérios para inclusão no presente estudo, foram registradas 36 espécies, sendo 10 de aves e 26 de mamíferos (Tabela 2), o que equivale a $72 \%$ das aves e $96 \%$ dos mamíferos de médio e grande porte registrados na Amazônia maranhense (Oren \& Roma, 2011; Lima et al., 2015; Vieira \& Oliveira 2020). Treze espécies (36\%) constam da lista oficial brasileira de espécies ameaçadas de extinção de acordo com a Portaria MMA 444/2014 (MMA, 2014; Tabela 2).

Tabela 1 - Esforço amostral (número de armadilhas-dia) e número de fotos de animais por período amostral do monitoramento TEAM na REBIO do Gurupi.

\begin{tabular}{|c|c|c|c|c|}
\hline Período amostral & $\begin{array}{c}\text { N. de armadilhas } \\
\text { fotográficas }\end{array}$ & Dias de campo & Esforço amostral & $\begin{array}{c}\text { N. de fotos de } \\
\text { animais }\end{array}$ \\
\hline 2016 & 61 & 43 & 2.623 & 25.008 \\
\hline 2017 & 61 & 70 & 4.270 & 28.581 \\
\hline 2018 & $58^{*}$ & 52 & 3.016 & 31.739 \\
\hline 2019 & 61 & 51 & 3.111 & 28.871 \\
\hline Total & & $\mathbf{2 1 6}$ & $\mathbf{1 3 . 0 2 0}$ & $\mathbf{1 1 4 . 1 9 9}$ \\
\hline
\end{tabular}

* 3 armadilhas tiveram seus registros perdidos. 
Tabela 2 - Lista das espécies de vertebrados de médio e grande porte registradas ao longo de 4 anos (20162019) na REBIO do Gurupi e seu status de ameaça de acordo com a lista oficial brasileira de espécies ameaçadas de extinção (MMA, 2014).

\begin{tabular}{|c|c|c|c|c|c|}
\hline Classe & Ordem & Família & Espécie & Nome comum & Status \\
\hline \multirow{33}{*}{ 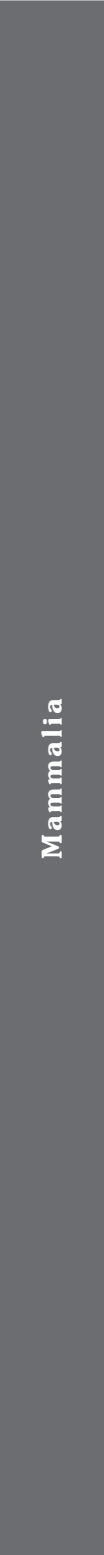 } & \multicolumn{5}{|l|}{ Carnivora } \\
\hline & & \multirow{2}{*}{ Canidae } & Cerdocyon thous & Cachorro-do-mato & \\
\hline & & & Speothos venaticus & Cachorro-vinagre & VU \\
\hline & & \multirow{6}{*}{ Felidae } & Leopardus pardalis & Jaguatirica & \\
\hline & & & Leopardus wiedii & Gato-maracajá & VU \\
\hline & & & Leopardus tigrinus & Gato-do-mato-pequeno & EN \\
\hline & & & Panthera onca & Onça-pintada & $\mathrm{VU}$ \\
\hline & & & Puma concolor & Puma, Onça-parda & VU \\
\hline & & & Puma yagouaroundi & Jaguarundi & VU \\
\hline & & \multirow{2}{*}{ Mustelidae } & Eira barbara & Irara, Papa-mel & \\
\hline & & & Galictis vittata & Furão & \\
\hline & & Procyonidae & Nasua nasua & Quati & \\
\hline & \multicolumn{5}{|l|}{ Pilosa } \\
\hline & & \multirow{2}{*}{ Myrmecophagidae } & Myrmecophaga tridactyla & Tamanduá-bandeira & VU \\
\hline & & & Tamandua tetradactyla & Tamanduá-mirim & \\
\hline & \multicolumn{5}{|l|}{ Cingulata } \\
\hline & & \multirow{5}{*}{ Dasypodidae } & Cabassous unicinctus & Tatu-rabo-de-couro & \\
\hline & & & Dasypus novemcinctus & Tatu & \\
\hline & & & Dasypus kappleri & Tatu-quinze-kilos & \\
\hline & & & Euphractus sexcinctus & Tatu-peba & \\
\hline & & & Priodontes maximus & Tatu-canastra & VU \\
\hline & \multicolumn{5}{|l|}{ Perissodactyla } \\
\hline & & Tapiriidae & Tapirus terrestris & Anta & VU \\
\hline & \multicolumn{5}{|l|}{ Artiodactyla } \\
\hline & & \multirow{2}{*}{ Cervidae } & Mazama americana & Veado-mateiro & \\
\hline & & & Mazama nemorivaga & Veado & \\
\hline & & \multirow{2}{*}{ Tayassuidae } & Pecari tajacu & Catitu & \\
\hline & & & Tayassu pecari & Porcão, Queixada & VU \\
\hline & \multicolumn{5}{|l|}{ Rodentia } \\
\hline & & Cuniculidae & Cuniculus paca & Paca & \\
\hline & & Dasyproctidae & Dasyprocta prymnolopha & Cutia & \\
\hline & \multicolumn{5}{|l|}{ Didelphimorphia } \\
\hline & & Didelphidae & Didelphis marsupialis & Gambá, Mucura & \\
\hline \multirow{13}{*}{$\stackrel{8}{8}$} & \multicolumn{5}{|l|}{ Galliformes } \\
\hline & & \multirow{3}{*}{ Cracidae } & Mitu tuberosum & Mutum-cavalo & \\
\hline & & & Penelope pileata & Jacupiranga & VU \\
\hline & & & Penelope superciliaris & Jacupemba & \\
\hline & & Odontophoridae & Odontophorus gujanensis & Uru-corcovado & \\
\hline & \multicolumn{5}{|l|}{ Gruiformes } \\
\hline & & Psophiidae & Psophia obscura & Jacamim-de-costas-escuras & $\mathrm{CR}$ \\
\hline & \multicolumn{5}{|l|}{ Tinamiformes } \\
\hline & & \multirow{5}{*}{ Tinamidae } & Crypturellus cirineus & Inhambu-pixuna & \\
\hline & & & Crypturellus soui & Sururina & \\
\hline & & & Crypturellus variegatus & Inhambu-anhangá & \\
\hline & & & Tinamus guttatus & Inhambu-galinha & \\
\hline & & & Tinamus tao & Azulona & $\mathrm{VU}$ \\
\hline
\end{tabular}

* Status de ameaça de extinção de acordo com a Lista Oficial Brasileira (Portaria MMA 444/2014): VU = Vulnerável; EN = em Perigo; CR = Criticamente em Perigo. 
A curva de acumulação de espécies apresentou tendência de estabilização, indicando que o levantamento para o grupo alvo está quase completo (Figura 3). Quanto à variação temporal na riqueza estimada de espécies, houve um aumento gradual, porém não significativo ao longo do período (Figura 4).

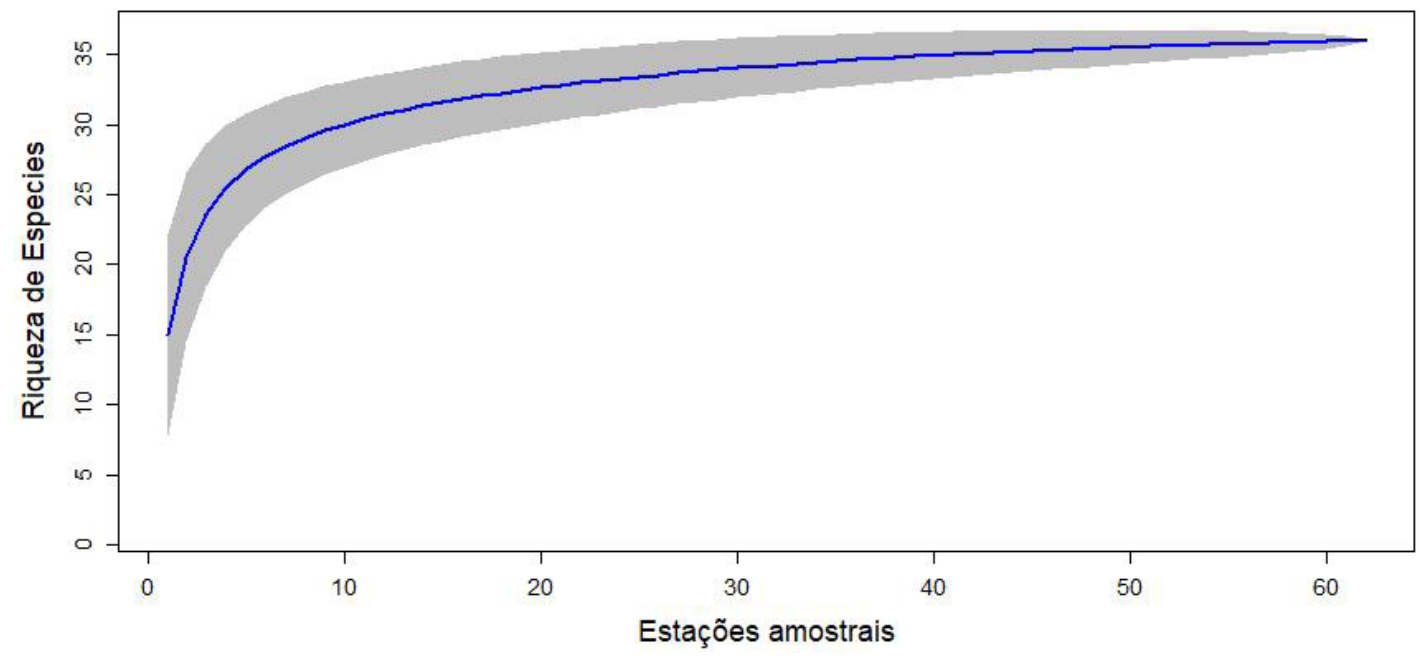

Figura 3 - Curva de acumulação de espécies monitoradas pelo protocolo TEAM realizado de 2016 a 2019 na REBIO do Gurupi/MA. Área sombreada indica o intervalo de confiança de 95\%. A curva foi gerada pelo método exact (Colwell et al., 2004).

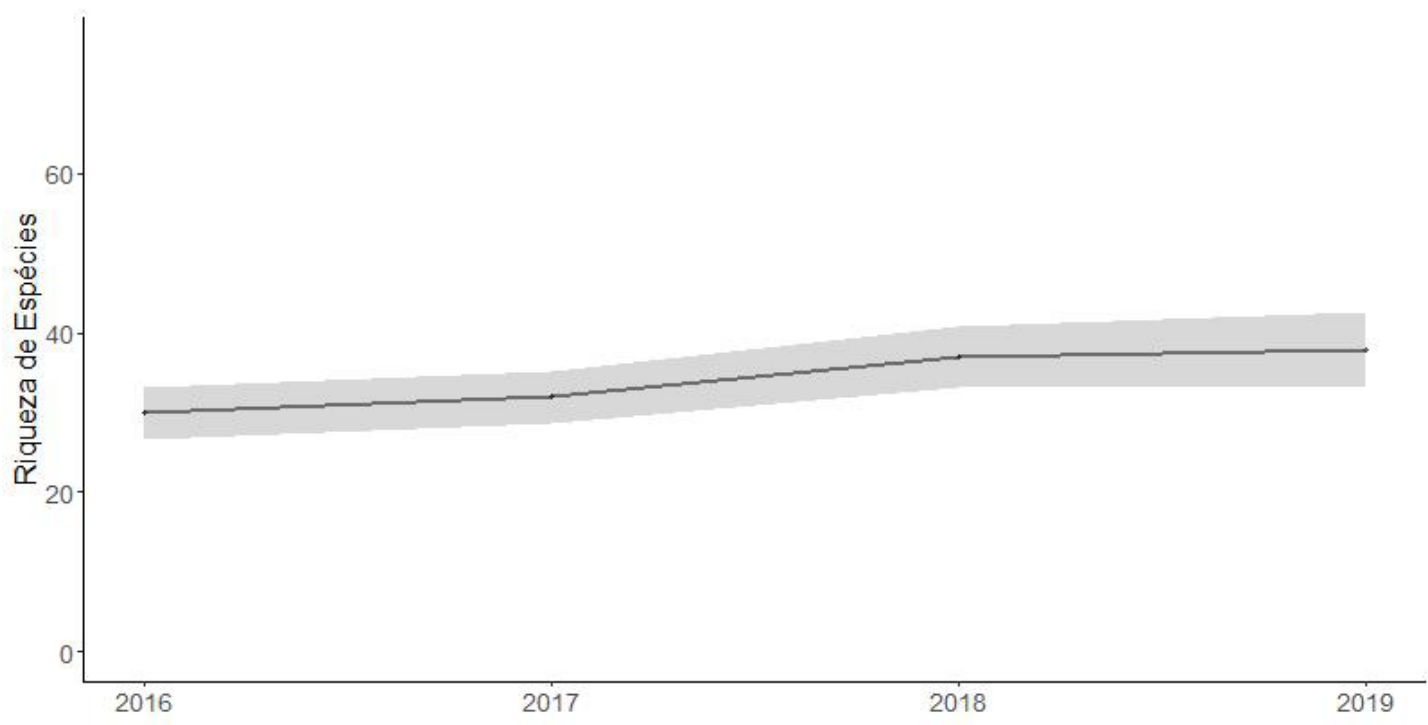

Figura 4 - Estimativa anual da riqueza de vertebrados terrestres de médio e grande porte na REBIO do Gurupi/MA, pelo estimador Jackknife 1 (Burnham \& Overton, 1978, 1979). Área sombreada indica o intervalo de confiança de $95 \%$.

Todas as espécies monitoradas apresentaram tendência de aumento na taxa da ocupação entre o primeiro e o último ano de monitoramento, e para a maioria delas essa tendência foi significativa (Figura 5). 


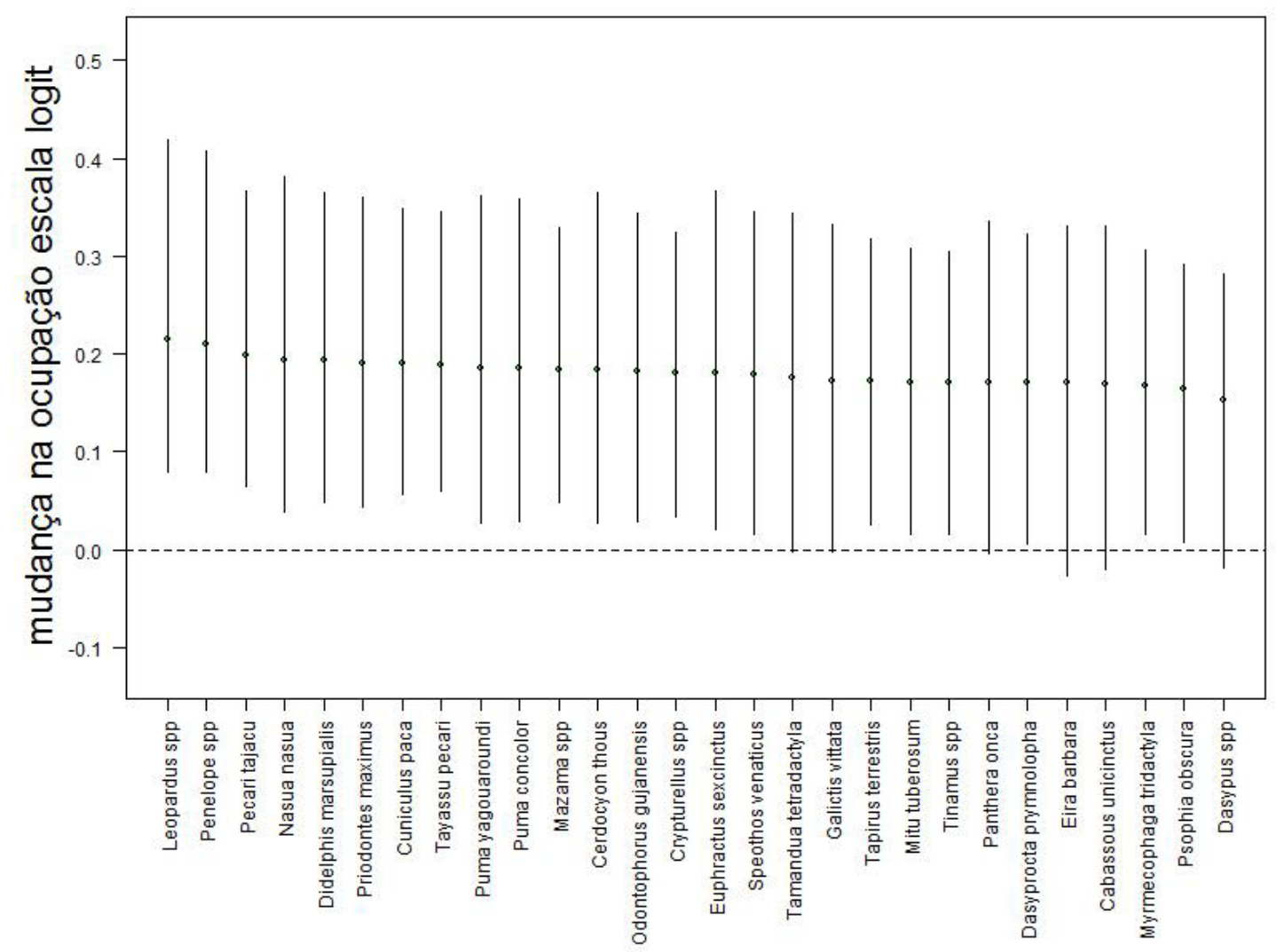

Figura 5 - Mudança na taxa de ocupação das espécies monitoradas na REBIO do Gurupi a partir do ano base 2016 (linha tracejada). As barras correspondem ao intervalo de confiança de $90 \%$.

Considerando somente as espécies ameaçadas de extinção, foram observadas taxas de ocupação relativamente mais altas para o puma Puma concolor, o tamanduá-bandeira Myrmecophaga tridactyla, e a anta Tapirus terrestris (Figura 6). As taxas de ocupação das espécies ameaçadas permaneceram relativamente estáveis ao longo dos quatro anos de monitoramento, com a notável exceção do jacamim-de-costas-escuras Psophia obscura, que oscilou consideravelmente entre 2016 e 2019 (Figura 6). É importante observar também que os intervalos de confiança foram muito largos para as espécies raras, o que dificulta inferências sobre suas tendências populacionais.

O WPI da comunidade aumentou significativamente ao longo do período de monitoramento. O aumento ocorreu entre o 2016 e 2017, permanecendo estável nos anos seguintes (Figura 7).

\section{Discussão}

A riqueza de vertebrados de médio $e$ grande porte observada na REBIO do Gurupi foi similar à riqueza conhecida para a Amazônia maranhense, indicando que a UC protege todo o complemento de espécies alvo deste estudo. Resultados semelhantes foram encontrados para as comunidades de primatas (Buss et al., 2017) e de aves (Lima et al., 2015), e pode ser justificado pelo contexto da paisagem, onde a REBIO do Gurupi e as terras indígenas contíguas formam um continuum de floresta amazônica capaz de manter populações viáveis ao longo do tempo (Oren \& Roma, 2011; Buss et al., 2017). Grandes fragmentos florestais são essenciais para manutenção da riqueza e da estrutura da comunidade de mamíferos (Ahumada et al., 2011). 

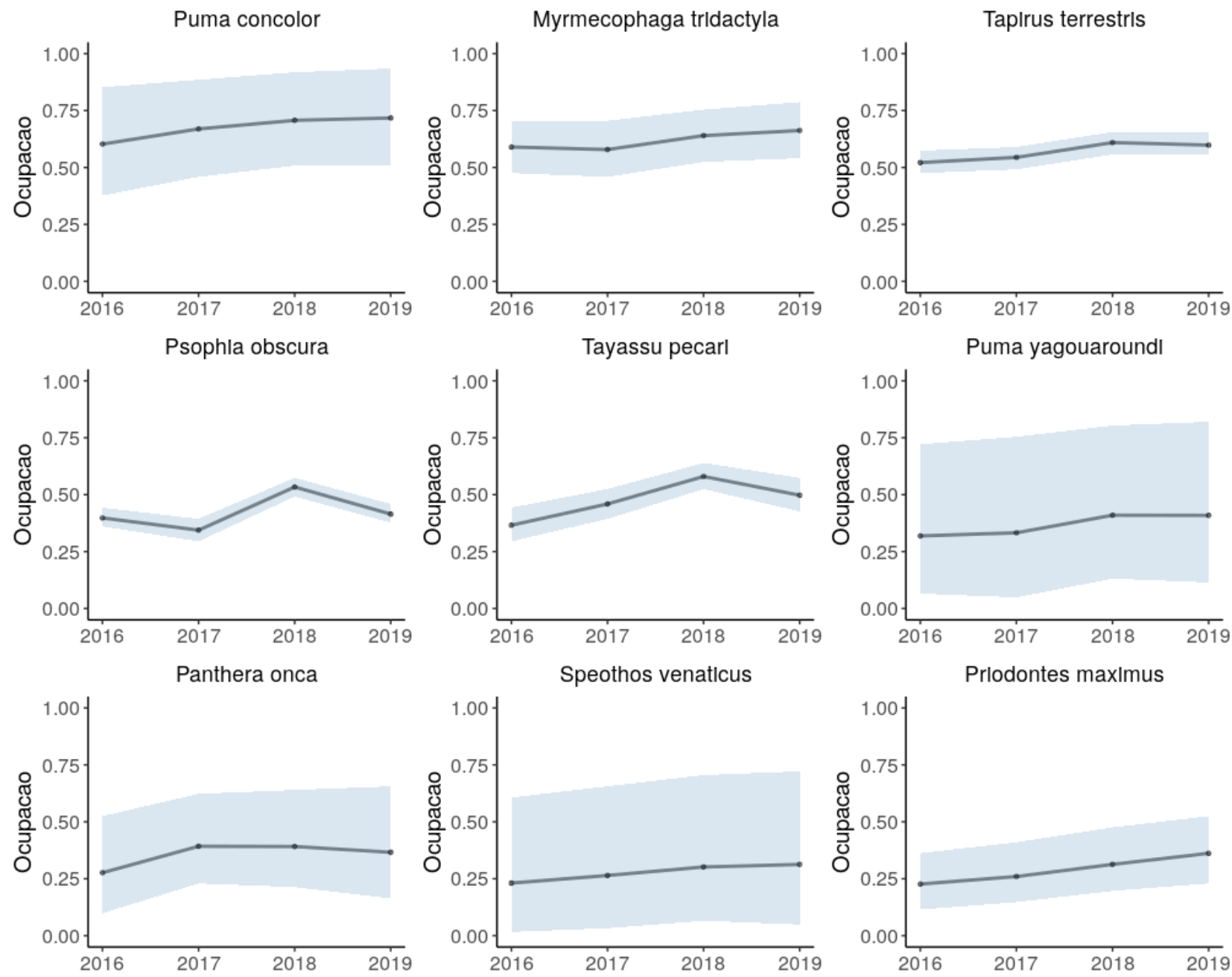

Figura 6 - Tendências temporais na taxa de ocupação de espécies ameaçadas de extinção monitoradas de 2016 a 2019 na REBIO do Gurupi. As áreas sombreadas indicam o intervalo de confiança de $90 \%$.

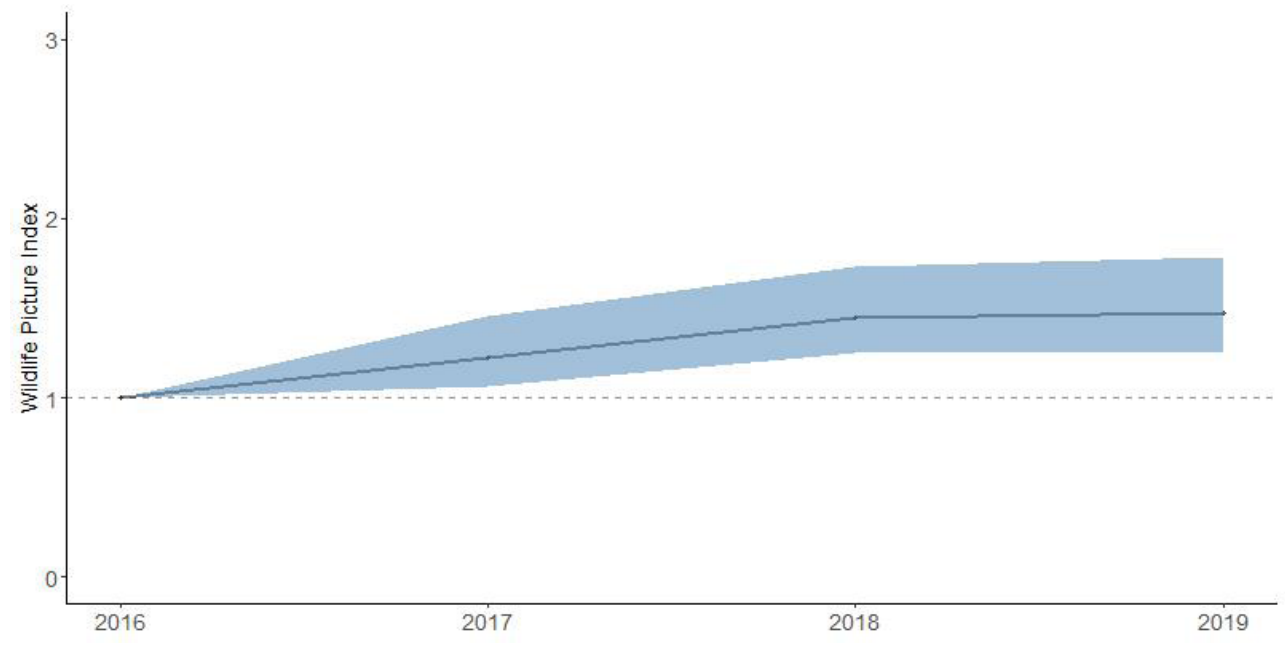

Figura 7 - Wildlife Picture Index para a Reserva Biológica do Gurupi avaliado no período de 2016 a 2019 de monitoramento. A área sombreada indica o intervalo de confiança de $90 \%$. 
A diversidade observada na REBIO do Gurupi se expressa não somente na riqueza de espécies, mas também pela variedade dos grupos tróficos (herbívoros, onívoros, carnívoros $e$ insetívoros), pela ampla variação na massa corporal $(0,5 \mathrm{a}>200 \mathrm{~kg})$ e pela redundância funcional, com múltiplas espécies utilizando recursos similares (e.g. puma e onça pintada). Essa diversidade é essencial para o funcionamento dos ecossistemas, como dispersão de sementes e controle de populações (Dirzo et al., 2014; Ripple et al., 2014; Osuri et al., 2016,), bem como para sua resiliência (Peterson et al., 1998; Gunderson, 2000). A presença de grandes carnívoros, como as onças, afeta direta e indiretamente a abundância de diversas outras espécies (Beschta \& Ripple, 2009; Estes et al., 2011). Grandes herbívoros, como as antas, são fundamentais na dispersão de grandes sementes a longas distancias, contribuindo para a manutenção da estrutura e composição da vegetação na paisagem local (O'Farrill et al., 2013). A coexistência de espécies que competem pelos mesmos recursos ecológicos indica a funcionalidade do ecossistema para a manutenção da biodiversidade (Chesson, 2000).

Na composição da comunidade também se destaca o alto percentual de espécies ameaçadas de extinção (36\%), o que já era esperado, pois vertebrados de médio e grande porte estão entre as espécies mais afetadas pela perda e fragmentação de habitat, caça e conflitos com atividades humanas (Schipper et al., 2008; Grooten \& Almond, 2018). As populações das espécies ameaçadas de extinção se mantiveram estáveis durante os quatro anos de monitoramento, demonstrando a importância da REBIO do Gurupi para alvos de conservação da biodiversidade, com destaque para o jacamim-decostas-escuras Psophia obscura, espécie endêmica, criticamente ameaçada de extinção conforme Portaria MMA 444/14 (MMA, 2014), e para a qual inexistem informações sobre sua biologia $e$ ecologia.

A riqueza de espécies e o alto número de espécies ameaçadas de extinção corroboram a indicação da região da REBIO do Gurupi como um hotspot global para conservação de mamíferos, devido ao alto nível de desmatamento observado em região de alta riqueza e com grande número de mamíferos ameaçados de extinção (Ceballos \& Ehrlich, 2006).

O aumento na taxa de ocupação, observado para a maior parte das espécies monitoradas, evidencia a saúde e resiliência do ecossistema na REBIO do Gurupi. A disponibilidade regular de populações de presas, como o cateto, queixada, veados, é um pré-requisito para a viabilidade de população de predadores topo de cadeia, como a onça-pintada (Sanderson et al., 2002; Astete et al., 2007). O tamanduá-bandeira, mirmecófago, grupo trófico geralmente mais sensível à perda de habitat e distúrbios ambientais (Ahumada et al., 2011; Rovero et al., 2020), teve uma das taxas de ocupação mais altas dentre as espécies ameaçadas de extinção. Os índices estáveis com uma alta significativa em 2018 da população de jacamim-de-costas-escuras, espécie endêmica e criticamente ameaçada de extinção, também merece destaque.

O WPI aumentou entre os anos de 2016 e 2019, como reflexo do aumento na taxa de ocupação da maior parte das espécies ao longo dos quatro anos de monitoramento, uma vez que o WPI é sensível a esse parâmetro (O'Brien et al., 2010). É possível que esse aumento tenha relação com o grande incêndio florestal que assolou a REBIO do Gurupi no final de 2015, pouco antes do início do monitoramento. Neste caso, a linha de base do primeiro ano de amostragem (2016) representaria uma situação atípica, pósimpacto, e o aumento e posterior estabilização do WPI indicaria um retorno da biota às condições anteriores. Independente da explicação, o aumento no WPI demonstra a resiliência da UC frente a distúrbios ambientais, e mostra que ela tem cumprido seu objetivo de preservar a fauna regional (Brasil, 1988).

Esses resultados positivos devem ser analisados considerando o contexto da paisagem, onde o grande fragmento de floresta contínua formado pela REBIO e terras indígenas, com mais de 2 milhões de hectares, possivelmente atenuaram os efeitos das queimadas, provendo refúgios e áreas fonte para recolonização após cessado o distúrbio (Leonard et al., 2014).

O monitoramento com armadilhas fotográficas também revelou ameaças à conservação que não foram identificadas pelos indicadores de biodiversidade analisados. Dois registros fotográficos, em anos distintos, detectaram cachorros-vinagre Speothos venaticus acometidos por doença de pele, possivelmente sarna sarcóptica (Jorge RSP, comunicação pessoal). Em um registro de 2019, uma matilha com 4 indivíduos estava doente (Figura 8). O cachorro-vinagre é espécie 
vulnerável (Portaria MMA 444/14), naturalmente rara e geralmente associada a ambientes florestais bem preservados (Jorge et al., 2013). Dentre as principais ameaças à conservação do cachorrovinagre estão as doenças (raiva, parvovirose, sarna sarcóptica) que podem ser adquiridas de animais domésticos (Jorge et al., 2008; Oliveira, 2009). Os registros fotográficos de cachorros-vinagre doentes refletem como as ocupações humanas na REBIO do Gurupi podem representar fontes de impacto à conservação da biodiversidade.

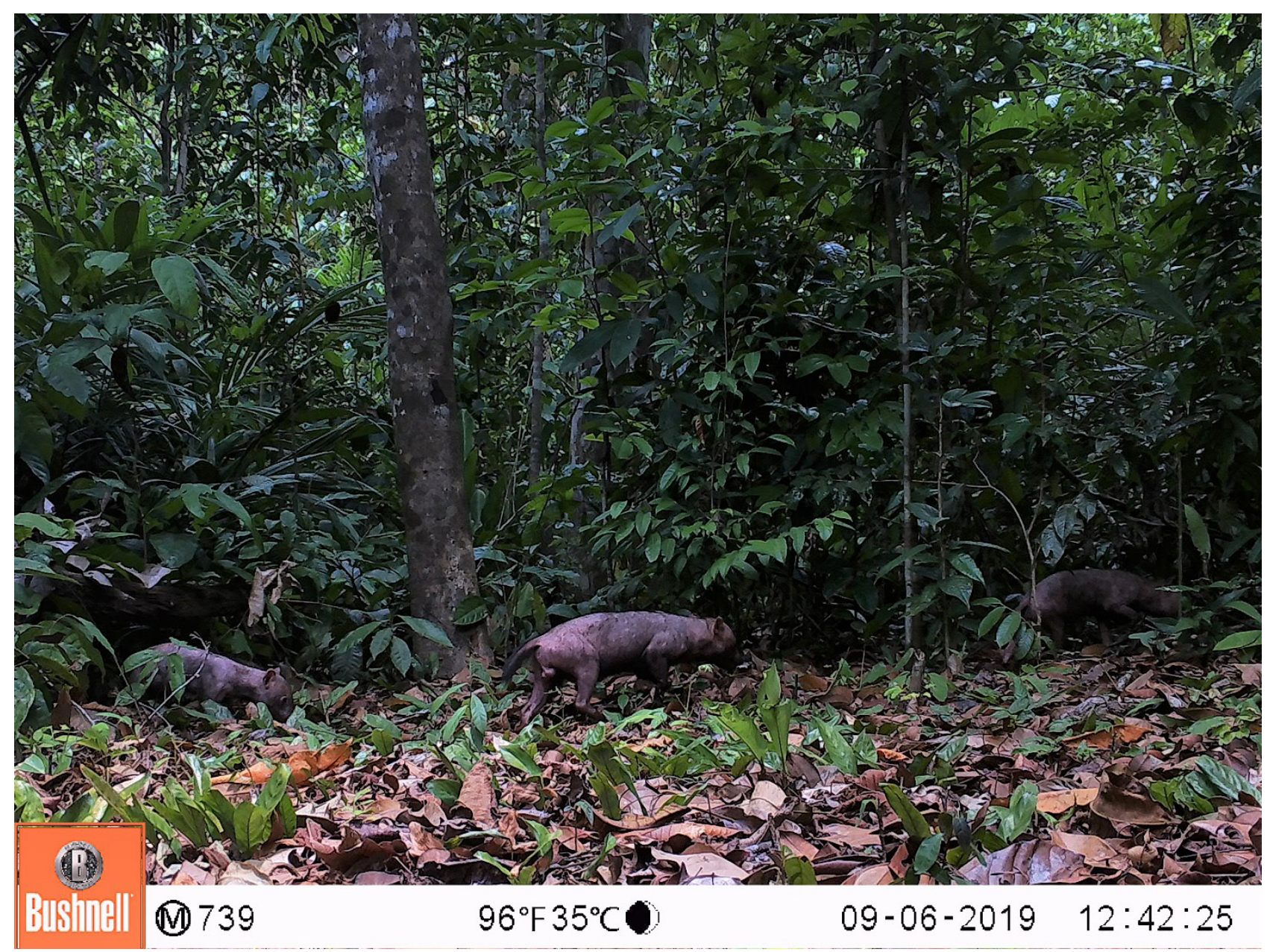

Figura 8 - Matilha de cachorro-vinagre com sarna sarcóptica registrada no monitoramento TEAM na REBIO do Gurupi em 2019. Acervo CENAP/ICMBio.

\section{Conclusões}

O protocolo TEAM gerou dados de alta qualidade sobre o estado de conservação das populações monitoradas, incluindo diversas espécies raras e difíceis de amostrar por outros métodos (e.g., onça-pintada Panthera onca, tatucanastra Priodontes maximus, cachorro-vinagre Speothos venaticus etc.). O WPI foi sensível às mudanças observadas nas populações $e$ a dinâmica observada foi consistente com o que se sabe sobre impactos recentes ocorridos, no caso dos incêndios florestais na REBIO do Gurupi. Os resultados obtidos até o momento demonstram que a REBIO do Gurupi tem sido efetiva em seu objetivo de manter a biodiversidade de vertebrados terrestres de médio e grande porte da Amazônia maranhense. As populações monitoradas, inclusive de espécies endêmicas e ameaçadas, permaneceram estáveis ao longo de 4 anos de monitoramento. Os resultados também indicam a capacidade de resiliência frente a eventos extremos como ocorreu na queimada de 2015. A REBIO do Gurupi tem um papel de destaque na 
conservação dos vertebrados terrestres de médio $e$ grande porte da Amazônia maranhense, porém os resultados positivos do monitoramento da REBIO do Gurupi devem ser observados considerando a paisagem onde está inserida, formando um continumm florestal com as terras indígenas.

Por outro lado, as ocupações e atividades humanas alheias aos objetivos de criação que ainda persistem no interior da UC, representam ameaças à conservação da biodiversidade. O registro de indivíduos de espécie ameaçada de extinção acometidos por doenças de pele possivelmente transmitidas por cachorros domésticos, acende o alerta para os impactos negativos das ocupações humanas no interior da REBIO do Gurupi, e representa um risco eminente para a subpopulação existente na UC. Esses registros destacam a importância do monitoramento e do desenvolvimento de pesquisas que possam melhor investigar os impactos humanos sobre a biodiversidade local.

Projeções científicas recentes sugerem que a Amazônia se encontra em fase de transição (Davidson et al., 2012), com possibilidade de mudanças significativas em seus padrões climáticos, cobertura florestal e produtividade. Nos cenários mais drásticos, a Amazônia passaria por um "ponto de virada" [tipping point] (Nobre \& Borma, 2009), com severas consequências para a região. Tudo isso reforça a importância $e$ necessidade de se manter o monitoramento de longo prazo, necessário tanto para detectar como para antever impactos sobre as áreas protegidas e sua biota. Desta forma, recomendamos que as atividades de monitoramento sejam mantidas para acompanhar as tendências da biodiversidade e subsidiar estratégias de intervenção e manejo que se fizerem necessárias. É recomendável que seja obtida uma série temporal de ao menos 10 anos (Field et al., 2007; Beaudrot et al., 2018). O próprio aumento na duração da série é suficiente para aumentar o poder estatístico e, consequentemente, a confiança nos resultados (Field et al., 2007).

\section{Agradecimentos}

O protocolo TEAM na REBIO do Gurupi é resultado do esforço coletivo de parceiros que contribuem para a realização anual dessa importante ferramenta de monitoramento da biodiversidade. Agradecemos à equipe de campo, em especial ao Abelha, à Ana Marta e Marília; aos gestores da REBIO do Gurupi, em especial à Evane, Luciana e ao Ivan Leão; e aos gestores do CENAP, em especial ao Ronaldo e Francisco Chen. Os códigos para o modelo multiespécies foram gentilmente cedidos por Jorge Ahumada (Conservação Internacional). A realização do protocolo TEAM na REBIO do Gurupi foi autorizada pelo órgão competente mediante licença SISBIO 55118, e conta com o apoio financeiro do Programa ARPA.

\section{Referências}

Ahumada JÁ et al. 2011. Community structure and diversity of tropical forest mammals: data from a global camera trap network. Philosophical Transactions of the Royal Society B: Biological Sciences, 366(1578), 27032711. https://doi.org/10.1098/rstb.2011.0115.

Ahumada JA, Hurtado J \& Lizcano D. 2013. Monitoring the Status and Trends of Tropical Forest Terrestrial Vertebrate Communities from Camera Trap Data: A Tool for Conservation. PLoS ONE 8: e73707. Public Library of Science.

Ahumada JA, O'Brien TG, Mugerwa B \& Hurtado J. 2016. Camera trapping as a monitoring tool at national and global levels. Pages 197-218 in F. Rovero and F. Zimmerman, editors. Camera Trapping for wildlife Research. Pelagic Publishing, Exeter, UK.

Alteff $\mathrm{EF}$ et al. 2019. The rarest of the rare: rediscovery and status of the critically endangered Belem Curassow, Crax fasciolata pinima (Pelzeln, 1870). Papéis Avulsos De Zoologia, 59, e20195946. https://doi. org/10.11606/1807-0205/2019.59.46.

Almeida AS \& Vieira ICG. 2010. Centro de Endemismo Belém: status da vegetação remanescente e desafios para a conservação da biodiversidade e restauração ecológica. Revista de Estudos Universitários. Sorocaba, SP, v. 36, n. 3, p. 95-111.

AlvaresCA,StapeJL,SentelhasPC, deMoraesGonçalves JL \& Sparovek G. 2013. Köppen's climate classification map for Brazil. Meteorologische Zeitschrift, 22(6): 711728. https://doi.org/10.1127/0941-2948/2013/0507.

Astete $\mathrm{S}$ et al. 2007. Comparative ecology of jaguars in Brazil. CAT News 9-14.

Beaudrot L et al. 2016. Standardized Assessment of Biodiversity Trends in Tropical Forest Protected Areas: The End Is Not in Sight. PLoS Biology 14: e1002357. Public Library of Science.

Beaudrot L, Ahumada JA, O'Brien TG, Jansen PA. 2018. Detecting tropical wildlife declines through camera- 
trap monitoring: an evaluation of the Tropical Ecology Assessment and Monitoring protocol. Oryx: 1-4.

Beschta RL \& Ripple WJ. 2009. Large predators and trophic cascades in terrestrial ecosystems of the western United States. Biological Conservation, 142(11): 2401-2414. $\quad$ https://doi.org/https://doi. org/10.1016/j.biocon.2009.06.015.

Braga PIS. 1979. Subdivisão fitogeográfica, tipos de vegetação, conservação e inventário florístico da floresta Amazônica. Supl. Acta Amazonica, 9: 53-80.

Brasil. 1988. Decreto n`95.614, de 11 de janeiro de 1988. https:/www.planalto.gov.br/ccivil_03/decreto/1980-1989/ D95614.htm. Acesso em: 20/06/2020.

Braz LC, Pereira JLG, Ferreira LV \& Thalês MC. 2017. A situação das áreas de endemismo da amazônia com relação ao desmatamento e às áreas protegidas. Boletim de Geografia, v. 34, n. 3, p. 45-62.

Burnham KP \& Overton WS. 1978. Estimation of the size of a closed population when capture probabilities vary among animals. Biometrika 65: 625-633.

Burnham KP \& Overton WS. 1979. Robust estimation of population-size when capture probabilities vary among animals. Ecology 60: 927-936.

Buss $G$ et al. 2017. Abundância e densidade de primatas na Reserva Biológica do Gurupi, Maranhão. Brasil. Biodiversidade Bras. 47-57.

Carvalho Jr. EAR. 2012. Relatório Anual de Atividades de Pesquisa e Monitoramento do Centro Nacional de Predadores/CENAP/ICMBio. Não publicado.

Carvalho Jr. EAR. 2017. Relatório de Avaliação populacional da onça-pintada na REBIO do Gurupi \& Avaliação do impacto de incêndios florestais sobre a fauna da REBIO do Gurupi. CENAP/ICMBio. Não publicado.

Carvalho Jr. EAR, Mendonça EN, Martins A \& Haugaasen T. 2020. Effects of illegal logging on Amazonian medium and large-sized terrestrial vertebrates. Forest Ecology and Management, 466: 118105. https://doi.org/https://doi.org/10.1016/j. foreco.2020.118105.

Ceballos G \& Ehrlich PR. 2006. Global mammal distributions, biodiversity hotspots, and conservation. Proceedings of the National Academy of Sciences, 103(51): 19374 LP - 19379. https://doi.org/10.1073/ pnas.0609334103.

Celentano D et al, 2017. Towards zero deforestation and forest restoration in the Amazon region of Maranhão state, Brazil. Land Use Policy 68: 692-698. https://doi. org/10.1016/j.landusepol.2017.07.041.

Chesson P. 2000. Mechanisms of maintenance of species diversity. Annu Rev Ecol Evol Syst 31: 343-366.
Annual Review of Ecology and Systematics. 31: 343-366. 10.1146/annurev.ecolsys.31.1.343.

Colwell R, Mao C \& Chang J. 2004 Interpolating, extrapolating, and comparing incidence-based species accumulation curves. Ecology 85: 2717-2727. Doi:10.1890/03-0557.

Davidson EA et al. 2012. The Amazon basin in transition. Nature 481: 321-328.

Dirzo R, Young HS, Galetti M, Ceballos G, Isaac NJB \& Collen B. 2014. Defaunation in the Anthropocene. Science, 345(6195): 401LP-406. https://doi.org/10.1126/science.1251817.

Estes JA et al. 2011. Trophic Downgrading of Planet Earth. Science, 333(6040): 301LP-306. https://doi. org/10.1126/science.1205106.

Fairweather PG. 1991. Statistical power and design requirements for environmental monitoring. Marine and Freshwater Research 42: 555-567.

Fegraus EH, Lin K, Ahumada JA, Baru C, Chandra S \& Youn C. 2011. Data acquisition and management software for camera trap data: A case study from the TEAM Network. Ecological Informatics 6: 345-353.

Field SA, O'Connor PJ, Tyre AJ \& Possingham HP. 2007. Making monitoring meaningful. Austral Ecology 32: 485-491.

Grooten M \& Almond REA. 2018. Living Planet Report. 2018: Aiming Higher. Gland, Switzerland.

Gunderson LH. 2000. Ecological Resilience - In Theory and Application. Annual Review of Ecology and Systematics, 31(1): 425-439. https://doi.org/10.1146/ annurev.ecolsys.31.1.425.

Hessel FO \& Lisboa EA. 2015. Mapa do estado de conservação da Reserva Biológica do Gurupi: Identificação das áreas conservadas e das áreas antropizadas. An. XVII Simpósio Bras. Sensoriamento Remoto - SBSR. 4224-4231.

IBAMA. 1999. Plano de Manejo da Reserva Biológica do Gurupi. IBAMA, Brasília. https:/www.icmbio.gov.br/ portal/component/content/article?id=1998:rebio-dogurupi. Acesso em: 20/08/2020.

IBGE. 2004. Mapa de Vegetação do Brasil. https:// mapas.ibge.gov.br/tematicos/vegetacao.html. Acesso em: 20/08/2020.

ICMBio. 2017. Instrução normativa n 03/2017. Brasília. https://www.icmbio.gov.br/portal/videos/24-legislacao/ instrucoes-normativas/115-instrucoes-normativas. Acesso em: 20/06/2020.

Jansen PA, Ahumada JA, Fegraus EH \& O'Brien TG. 2014. TEAM: a standardised camera trap survey to monitor terrestrial vertebrate communities in tropical 
forests. Pages 263-270. In: Meek P et al, (eds.) Camera trapping: wildlife management and research. CISRO, Collingwood, Australia.

Jorge RSP, Lima ES \& Lucarts LEB. 2008. Sarna sarcóptica ameaçando cachorros-vinagres (Speothos venaticus) de vida livre em Nova Xavantina/MT. In: Anais do XXXIII Congresso Anual da Sociedade de Zoológicos do Brasil. Disponível em https://www. biofaces.com/upload/post/2015/07/1435957325.pdf. Acesso em: 20/06/2020.

Jorge RSP, Beisiegel BM, Lima ES, Jorge MLSP, LeitePitman MRP \& Paula, RC. 2013. Avaliação do risco de extinção do Cachorro-vinagre Speothos venaticus (Lund, 1842) no Brasil. Revista Biodiversidade Brasileira, 3(1): 179-190.

Lacher TE et al. 2019. The functional roles of mammals in ecosystems. Journal of Mammalogy 100: 942-964.

Legg CJ \& Nagy L. 2006. Why most conservation monitoring is, but need not be, a waste of time. Journal of Environmental Management 78: 194-199.

Leonard SWJ, Bennett AF \& Clarke MF. (2014). Determinants of the occurrence of unburnt forest patches: Potential biotic refuges within a large, intense wildfire in south-eastern Australia. Forest Ecology and Management, 314: 85-93. https://doi.org/https://doi. org/10.1016/j.foreco.2013.11.036.

Lima DM, Martínez C \& Raíces DSL. 2015. An avifaunal inventory and conservation prospects for the Gurupi Biological Reserve, Maranhão, Brazil. Revista Brasileira de Ornitologia - Brazilian Journal of Ornithology. 22(4): 317-340.

MacKenzie DI, Nichols JD, Lachman GB, Droege S, Royle JA \& Langtimm CA. 2002. Estimating site occupancy rates when detection probabilities are less than one. Ecology 83: 2248-2255. John Wiley e Sons, Ltd.

MacKenzie DI, Nichols JD, Hines JE, Knutson MG \& Franklin AB. 2003. Estimating site occupancy, colonization, and local extinction when a species is detected imperfectly. Ecology 84: 2200-2207.

MacKenzie DI \& Nichols JD. 2004. Occupancy as a surrogate for abundance estimation. Anim Biodivers Conserv 27: 461-467.

Martins MB \& Oliveira TG. 2011. Amazônia Maranhense diversidade e conservação. Museu Paraense Emílio Goeldi, Belem/PA. 328p.

MMA. 2014. Portaria 444 de 17 de dezembro de 2014. Diário Oficial da União. https:/www.mma.gov. br/biodiversidade/conservacao-de-especies/faunaameacada/fauna.html. Acesso em: 20/05/2020.

Monitora et al. 2018. Monitora: Programa nacional de monitoramento da biodiversidade. Subprograma
Terrestre Componente Florestal Relatório triênio 20142016. ICMBio, Brasília.

Nobre CA \& Borma LDS. 2009. "Tipping points" for the Amazon forest. Current Opinion in Environmental Sustainability 1: 28-36.

O'Brien TG, Baillie JEM, Krueger L \& Cuke M. 2010. The wildlife picture index: monitoring top trophic levels: the wildlife picture index. Anim. Conserv. 13: 335-343. http://dx.doi.org/10.1111/j.1469-1795.2010.00357.x.

O'Brien TG \& Kinnaird MF. 2013. The Wildlife Picture Index: A Biodiversity Indicator for Top Trophic Levels. Pages 45-70. In: Collen B et al. (eds.) Biodiversity Monitoring and Conservation: Bridging the Gap between Global Commitment and Local Action. John Wiley e Sons, Ltd, West Sussex, UK.

O'Farrill G, Galetti M \& Campos-Arceiz A. 2013. Frugivory and seed dispersal by tapirs: An insight on their ecological role. Integrative zoology. 8: 4-17. 10.1111/j.1749-4877.2012.00316.x.

Oliveira TG. 2009. Distribution, habitat utilization and conservation of the Vulnerable bush dog Speothos venaticus in northern Brazil. Oryx, 43: 247-25.

Oksanen $\mathrm{J}$ et al. 2019. The Vegan Package, version 2: 5-6. https:/cran.r-project.org/web/packages/vegan/ vegan.pdf. Acesso em: 20/05/2020.

Oren DC \& Roma JC. 2011. Composição e vulnerabilidade da avifauna da Amazônia maranhense, p. 221-247. In: Martins MB \& Oliveira TG (eds.). Amazônia Maranhense: Diversidade e Conservação. Belém: MPEG.

Osuri A et al. 2016. Contrasting effects of defaunation on aboveground carbon storage across the global tropics. Nat Commun 7: 11351. https://doi.org/10.1038/ ncomms 11351 .

Peterson G, Allen C \& Holling C. 1988. Ecological Resilience, Biodiversity, and Scale. Ecosystems 1: 6-18. https://doi.org/10.1007/s100219900002.

Plummer M (2003). JAGS: A program for analysis of Bayesian graphical models using Gibbs sampling. Proceedings of the 3rd International Workshop on Distributed Statistical Computing (DSC 2003). March: 20-22.

R Development Core Team. 2019. R: A language and environment for statistical computing. R Foundation for Statistical Computing, Vienna, Austria.

Ripple WJ et al. 2014. Status and Ecological Effects of the World's Largest Carnivores. Science, 343(6167): 1241484. https://doi.org/10.1126/science.1241484.

Rovero F \& Ahumada J. 2017. The Tropical Ecology, Assessment and Monitoring (TEAM) Network: An early warning system for tropical rain forests. Science of the 
Total Environment, 574(September): 914-923. https:// doi.org/10.1016/j.scitotenv.2016.09.146.

Rovero $\mathrm{F}$ et al. 2020. A standardized assessment of forest mammal communities reveals consistent functional composition and vulnerability across the tropics. Ecography, 43(1): 75-84. https://doi. org/10.1111/ecog.04773.

Sanderson EW et al. 2002. Planning to save a species: the jaguar as a model. Conserv. Biol. 16: 58-72.

Schipper $\mathrm{J}$ et al. 2008. The Status of the World's Land and Marine Mammals: Diversity, Threat, and Knowledge. Science 322: 225-230.

Silva JMC, Rylands AB \& Fonseca GAB. 2005. $\mathrm{O}$ destino das áreas de endemismo da Amazônia. Megadiversidade, 1: 1-124-131.

Sollmann R. 2018. A gentle introduction to camera-trap data analysis. African Journal of Ecology 56: 740-749.
TEAM Network. 2011. Terrestrial vertebrate (camera trap) monitoring protocol implementation manual. Page Terrestrial Vertebrate Protocol Implementation Manual.

Wilman H, Belmaker J, Simpson J, de la Rosa C, Rivadeneira MM \& Jetz W. 2014. EltonTraits 1.0: Species evel foraging attributes of the world's birds and mammals. Ecology, 95: 2027-2027. doi:10.1890/131917.1.

Vieira OQ \& Oliveira TG. 2020. Non-volant mammalian species richness in the ecotonal Brazilian midnorth: checklist for Maranhão State. Biota Neotropica 20(2): e20190912. https://doi. org/10.1590/1676-0611-BN-2019-0912.

Yu-Sung S. 2020. R2jags: A Package for Running jags from R. Package version 0: 6-1. CRAN. https://cran.rproject.org/web/packages/R2jags/R2jags.pdf. Acesso em: 20/06/2020.

\section{Biodiversidade Brasileira - BioBrasil. \\ Fluxo Contínuo}

n. 3, 2021

http://www.icmbio.gov.br/revistaeletronica/index.php/BioBR

Biodiversidade Brasileira é uma publicação eletrônica científica do Instituto Chico Mendes de

Conservação da Biodiversidade (ICMBio) que tem como objetivo fomentar a discussão e a disseminação de experiências em conservação e manejo, com foco em unidades de conservação $e$ espécies ameaçadas.

ISSN: 2236-2886 